\title{
Progenitors of electron-capture supernovae
}

\author{
Samuel Jones ${ }^{1, *}$, Raphael Hirschi ${ }^{1,2}$, Falk Herwig ${ }^{3}$, Bill Paxton $^{4}$, \\ Francis X. Timmes ${ }^{5,6}$ and Ken'ichi Nomoto ${ }^{2}$ \\ ${ }^{1}$ Astrophysics Group, Lennard Jones Building, Keele University ST5 5BG, UK \\ ${ }^{2}$ Kavli IPMU, University of Tokyo, Kashiwa, Chiba 277-8583, Japan \\ ${ }^{3}$ Department of Physics and Astronomy, Victoria, BC V8W 3P6, Canada \\ ${ }^{4}$ KITP and Dept. of Physics, University of California, Santa Barbara, CA 93106 USA \\ ${ }^{5}$ Joint Institute for Nuclear Astrophysics, University of Notre Dame, IN 46556, USA \\ ${ }^{6}$ School of Earth and Space Exploration, University of Arizona, Tempe, AZ 85287, USA \\ *email: s.w.jones@epsam.keele.ac.uk
}

\begin{abstract}
We investigate the lowest mass stars that produce Type-II supernovae, motivated by recent results showing that a large fraction of type-II supernova progenitors for which there are direct detections display unexpectedly low luminosity (for a review see e.g. Smartt 2009). There are three potential evolutionary channels leading to this fate. Alongside the standard "massive star' Fe-core collapse scenario we investigate the likelihood of electron capture supernovae (EC$\mathrm{SNe}$ ) from super-AGB (S-AGB) stars in their thermal pulse phase, from failed massive stars for which neon burning and other advanced burning stages fail to prevent the star from contracting to the critical densities required to initiate rapid electron-capture reactions and thus the star's collapse. We find it indeed possible that both of these relatively exotic evolutionary channels may be realised but it is currently unclear for what proportion of stars. Ultimately, the supernova light curves, explosion energies, remnant properties (see e.g. Knigge et al. 2011) and ejecta composition are the quantities desired to establish the role that these stars at the lower edge of the massive star mass range play.
\end{abstract}

Keywords. stars: evolution, supernovae: general

\section{Preliminary models}

For stars that develop degenerate $\mathrm{ONe}$ cores with $M_{\mathrm{ONe}} \gtrsim 1.37 M_{\odot}$, neon is ignited off-centre. Such an ignition was also found in the models of Nomoto (1984), but the subsequent evolution was not followed to a conclusion. As we discuss below, the nucleosynthesis in these neon-burning shells becomes rather complex and the speed and nature of their inward propagation determine the evolutionary outcome.

We computed a $9 M_{\odot}$ model from pre-main sequence using the MESA code (Paxton et al. 2011). Following the main sequence and core He-burning, our model ignites carbon non-degenerately at its centre where the stellar material becomes convectively unstable, which is typical of a massive star with $M_{\text {ini }} / M_{\odot} \lesssim 20$. Following the ignition of convective secondary carbon burning shells, neon is ignited at the co-ordinate $\sim 0.75 M_{\odot}$ away from the centre. Ignition takes place where the maximum temperature now resides due to a temperature inversion in the core caused by the onset of degeneracy following the central extinction of carbon.

The centre contracts since there is no constant source of energy production there, only neutrino losses and URCA cooling. Meanwhile, energy production in the shell pushes the temperature there high enough to ignite oxygen, leaving behind mostly isotopes of $\mathrm{Si}$ and $\mathrm{S}$. We find that the shell propagates by means of compressional heating, when 
gravitational energy released in contraction raises the temperature of the matter to the point of ignition. For that reason, in 1-dimension the propagation is seen as a series of flashes then contraction following extinction of the previous flash event.

There are two important timescales involved after neon is ignited: $\tau_{\text {flame }}$, the timescale in which the propagating neon-oxygen burning shell will reach the centre and $\tau_{\rho}$, the timescale in which the centre will contract to the threshold density critical for electrons to begin to capture on ${ }^{20} \mathrm{Ne}$ nuclei.

In our $9 M_{\odot}$ model, $\tau_{\rho}<\tau_{\text {flame }}$ and the central density surpasses the threshold for electron captures on ${ }^{24} \mathrm{Mg}$ to become energetic when the base of the flame is still $\sim 0.7 M_{\odot}$ out from the centre. Assuming the Ledoux stability criterion for convection, the temperature peaks in the $\rho_{\mathrm{c}}-T_{\mathrm{c}}$ plane at locations corresponding to the threshold densities for electron captures on ${ }^{24} \mathrm{Mg}$ and ${ }^{20} \mathrm{Ne}$ as seen in Miyaji \& Nomoto (1987) for example as the core contracts to higher densities. The central temperature begins to increase due to ${ }^{20} \mathrm{Ne}+e^{-}$at $\log _{10}\left(\rho_{\mathrm{c}}\left(\mathrm{g} \mathrm{cm}^{-3}\right)\right)=9.92$, eventually rising sufficiently to ignite oxygen.

The density at which oxygen is ignited (and thus, the critical density for electron captures on ${ }^{20} \mathrm{Ne}$ ) determines whether the star will collapse or explode. For this reason it is crucial to include Coulomb corrections to the electron chemical potential, $\mu_{\mathrm{e}}$, and to the rate, both of which raise the effective threshold density (Gutierrez et al. 1996).

Lastly, while a S-AGB progenitor will have undergone a period of extended mass loss on the TP-SAGB, these low-mass massive stars for which $\tau_{\rho}<\tau_{\text {flame }}$ will have the majority of their hydrogen-rich envelope intact at the point of collapse, perhaps with interesting consequences for the SN light curve.

\section{Implications}

Our models imply that there may be an additional evolutionary channel producing electron-capture supernovae. In order to have an idea of the statistical contribution of these stars to observed supernovae, numerical simulations with the most up-to-date microphysics must be computed. Since the neon-oxygen burning shell propagates by compressional heating in a degenerate regime, a large network involving many weak processes in addition to key URCA process reactions must be included in order to follow properly the $Y_{\mathrm{e}}$ profile.

We expect that the transition from S-AGB to massive star is not a finite jump, but instead is rather continuous, having some interesting nucleosynthetic and evolutionary consequences. What we would like to know is for what proportion of stars is neon ignited off-centre, for what proportion of those stars is the condition for EC-SN, $\tau_{\rho}<\tau_{\text {flame }}$, satisfied; and if there are identifiable observational signatures that would enable us to differentiate between the different progenitor scenarios.

\section{References}

Gutierrez, J., Garcia-Berro, E., \& Iben, Jr. et al. 1996, ApJ, 459, 701

Knigge, C., Coe, M. J., \& Podsiadlowski, P. 2011, Nature, 479, 372

Miyaji, S. \& Nomoto, K. 1987, ApJ, 318, 307

Nomoto, K. 1984, ApJ, 277, 791

Paxton, B., Bildsten, L., Dotter, A., Herwig, F., Lesaffre, P., \& Timmes, F. 2011, ApJS, 192, 3

Smartt, S. J. 2009, ARAA, 47, 63 